\title{
Essais
}

ESSAIS

Revue interdisciplinaire d'Humanités

$8 \mid 2016$

Erreur et création

\section{L'erreur à l'œuvre et ses enjeux artistiques. Le cas chinois de la période maoïste à la période actuelle}

Frédéric Le Gouriérec

\section{(2) OpenEdition}

1 Journals

Édition électronique

URL : http://journals.openedition.org/essais/5302

DOI : 10.4000/essais.5302

ISSN : 2276-0970

Éditeur

École doctorale Montaigne Humanités

Édition imprimée

Date de publication : 15 mars 2016

Pagination : 142-156

ISBN : 978-2-9544269-7-6

ISSN : $2417-4211$

Référence électronique

Frédéric Le Gouriérec, « L'erreur à l'œuvre et ses enjeux artistiques. Le cas chinois de la période maoïste à la période actuelle », Essais [En ligne], 8 | 2016, mis en ligne le 28 octobre 2020, consulté le 30 octobre 2020. URL : http://journals.openedition.org/essais/5302 ; DOI : https://doi.org/10.4000/ essais.5302 


\section{L'erreur à l'œuvre et ses enjeux artistiques. Le cas chinois de la période maoïste à la période actuelle}

\section{Frédéric Le Gouriérec}

\section{Préambule sur l'art lettré : de la nature de l'Art}

La notion d'erreur est a priori incompatible avec le fondement même de la tradition artistique chinoise qu'est la peinture lettrée, née sous les Song (9601279) d'une opposition frontale à la réglementation propre à la peinture de cour. La distance culturelle qui sépare l'observateur moderne, chinois comme étranger, de la source de ces deux traditions critiques distinctes ne fait qu'accroître la confusion introduite par la proximité sociologique, effectivement avérée pendant des siècles, entre les artistes pratiquant l'un ou l'autre de ces deux genres, mais parfois aussi les deux ; entérinée, en dépit du flagrant contreexemple contemporain, par des collectionneurs moins clairvoyants que n'est tenue de le croire l'historiographie conventionnelle, démunie sans ce présupposé, cette confusion n'est pas près non plus d'être dissipée par le discours vaporeux toléré si ce n'est encouragé dans le domaine de «l'esthétique chinoise ».

La peinture de cour est une peinture académique qui se fait un point d'honneur, tout en produisant de la belle ouvrage, d'accomplir un travail en adéquation avec la fonction qui lui est assignée. En matière d'imagerie, les paramètres sur lesquels le jugement est sollicité peuvent être précisément définis. Une peinture est une réalisation technique, qui implique une sélection de matériaux et des procédés permettant de les agencer de façon à atteindre une fin déterminée. Cette fin relève de la représentation perçue et conçue : de fait, une peinture n'est ni plus ni moins qu'une machine à faire voir et comprendre. Reste donc à choisir ce qui mérite d'être vu et de quelle manière le thème doit être présenté. Définie en des termes si terre à terre, la peinture n’a déjà pas de quoi enthousiasmer des figures d'artistes farouches ou excentriques comme l'histoire de la peinture lettrée en propose à foison, à l'image d'un Xu Wei (1521-1593) sous les Ming ou d'un Badashanren (1625-1705) sous les Qing. Et c'est encore plus vrai lorsqu'une réglementation codifiée impose une orientation concrète aux choix possibles dans le cadre de ladite définition. 
Une peinture de cour n'a de valeur que si elle est réalisée avec des matières de premier ordre, forcément onéreuses, et à l'aide d'une technique minutieuse garantissant la restitution réaliste de l'objet de la représentation, dans laquelle nul élément extérieur ne viendrait interférer, surtout s'il marque l'empreinte de la petite personne de son exécutant. Du reste, seuls mériteraient d'être représentés les thèmes choisis pour leur importance réelle ou pour celle de leur commanditaire. In fine, la hiérarchie des thèmes aboutirait ainsi à privilégier ce qui sous d'autres climats s'appellerait la " peinture d'histoire " et en particulier le portrait des puissants, étendu à la figuration de leurs attributs et centres d'intérêt réels ou imaginaires. Pour finir, il faudrait encore que cette imagerie s'ingénie à magnifier son objet : voilà qui commence à faire beaucoup pour des lettrés en rupture de ban, dégoûtés des compromissions de la cour et loin d'être toujours fortunés ni même versés dans les derniers acquis techniques pourtant à la portée d'honnêtes artisans bien plus pragmatiques. Cette morale de la maîtrise, tournée vers l'application des règles définissant la tâche à exécuter, ne saurait être la leur. La peinture lettrée a ses propres règles, mais elles ne visent pas à l'accomplissement d'une tâche, elles sont l'occasion d'un jeu de contrastes ou d'échos entre des restrictions librement consenties sur tel paramètre de l'image et des débordements audacieux sur tel autre, sans la moindre visée pratique au-delà de cet exercice de liberté auto-suffisant. Les matières sont plus modestes, les gammes de couleurs très restreintes, la surface peinte parfois très limitée ; le thème choisi, n'en déplaise aux gloses philosophantes dont l'assise est souvent pur jeu de traduction, se doit de n'avoir aucune valeur en soi, si bien que montagne et eau, fleurs et oiseaux font très bien l'affaire. Mais à toutes ces frustrations délibérées et variablement dosées peut répondre une liberté virtuose du pinceau et de la touche, nullement assujettie à la restitution réaliste de l'objet. La peinture n'est qu'un prétexte et seul compte l'état d'esprit à l'œuvre au moment de sa confection, qui en recèle la trace ; la personnalité de l'auteur constitue elle-même l'un des critères entrant en jeu dans l'appréciation de l'œuvre, pour cette raison encore moins réductible à sa seule dimension matérielle.

Si l'erreur et la faute sont un écueil toujours possible dans le domaine de l'imagerie réglementée académique, comme ils le sont également dans l'imagerie populaire, leur place dans la culture lettrée paraît d'emblée beaucoup moins envisageable.

\section{La recherche de l'erreur et sa correction}

De même que la santé, pourtant éprouvée physiquement, ne se comprend que par la pathologie, qui en dévoile les ressorts insoupçonnés grâce à l'étude de leur subite défaillance, de même la nature d'un art se perçoit moins dans le discours descriptif ou théorique qui postule son évidence, que dans sa 
confrontation à un art autre qui, au nom de sa réglementation, lui impose des corrections non pas dites, tant le langage peut s'avérer imprécis, mais effectives. Ces moments rares de l'histoire de l'art font ressortir beaucoup plus clairement les fondements respectifs des genres concernés et le cas des peintres jésuites employés à la cour des Qing mérite une attention particulière.

Dans une lettre de 1743, Jean-Denis Attiret, en poste depuis 1738, se montre très clair : "Les portraits de l'Empereur et des Impératrices avaient été peints avant mon arrivée, par un de nos frères, nommé Castiglione, peintre italien, et très habile, avec qui je suis tous les jours. Tout ce que nous peignons est ordonné par l'Empereur. Nous faisons d'abord les dessins : il les voit, il les fait changer, réformer comme bon lui semble. Que la correction soit bien ou mal, il en faut passer par là sans oser rien dire $»^{1}$. En 1769, peu après la mort d'Attiret, le Père Jean-Matthieu Tournu de Ventavon dresse un tableau tout aussi tranché, récapitulant l'expérience des peintres jésuites à la cour :

Un peintre européen est au commencement bien embarrassé : il faut qu'il renonce à son goût et à ses idées sur bien des points, pour s'accommoder à celles du pays, et il n’y a pas moyen de faire autrement. Il faut même, tout habile qu'il peut être, qu'il devienne apprenti à certains égards. Ici dans les tableaux on ne veut point d'ombres, ou si peu que rien; c'est à l'eau que se font presque toutes les peintures; très peu sont à l'huile. Les premières en ce genre qu'on présenta à l'Empereur furent faites, dit-on, sur des toiles et avec des couleurs mal préparées. Peu de temps après, elles noircirent de façon à déplaire à l'Empereur, qui n'en veut presque plus. Enfin, il faut que les couleurs soient unies, et les traits délicats comme dans une miniature. Je n'ajoute pas mille autres circonstances qui ne laissent pas d'exercer la patience d'un nouveau venu. ${ }^{2}$

Ce dont il est question dans ces deux passages, c'est de l'académisme des uns passé au crible de l'académisme des autres : la mention de portraits impériaux n'aurait pas lieu d'être en peinture lettrée. Les capacités techniques des jésuites intéressent les Chinois dans le seul cadre de la peinture académique, mais il faut encore tout corriger tant les erreurs sont nombreuses selon le goût chinois : matériau fautif (l'huile des uns contre l'eau des autres), mauvais agencement des unités dans la fabrication de l'ouvrage (touche accumulée des uns contre couleurs unies et traits de miniature des autres), mauvaise conception du dessin, ajout fautif des ombres et " mille autres circonstances". Il n'est toutefois pas anodin que la radicalité de la correction des erreurs contestées in petto par les jésuites, en raison de l'arbitraire de cette divergence normative, trouve sa justification originelle dans une erreur interne à leur propre système technique qu'ils admettent eux-mêmes, celle d'un accident de préparation des matériaux essentiels, qui avait éveillé la défiance de l'Empereur et conditionné la suite des événements.

1 Lettres édifiantes et curieuses de Chine par des missionnaires jésuites 1702-1776, anthologie établie par Isabelle et Jean-Louis Vissière, Garnier-Flammarion, Paris, 1979, p. 426.

2 Ibid., p. 466. 
La célèbre sentence de Zou Yigui, qui avait été l'élève de Castiglione, montre que, de part et d'autre, chacun était conscient de l'apport des jésuites et savait l'analyser clairement dès lors qu'il était question de la seule technique et de la vision du monde qu'elle produisait. En revanche, le malentendu persistait sur la nature et l'ampleur de la faute profonde ; force est de reconnaitre que le caractère elliptique du raisonnement comme de la formulation des Chinois n'aidait pas à combler le fossé.

Les Occidentaux maîtrisent parfaitement la géométrie : ils peignent avec des oppositions de clair et d'obscur, de plans rapprochés ou éloignés, sans le moindre défaut. Les personnages, les bâtiments, les arbres qu'ils peignent ont tous une ombre portée. Dans ces peintures, les couleurs et le tracé du pinceau sont radicalement différents de ce qui se fait en Chine. La composition part du cadre général et se resserre, la proportion se mesurant par des triangles. Ils peignent les palais et les salles avec les murs, si bien qu'on a presque envie d'y entrer. Quand on les étudie, on peut leur emprunter des petites choses : ce ne sont que des façons d'éveiller son attention. Mais le travail du pinceau n'a pas la moindre règle ; malgré l'habileté, ce n'est que de l'artisanat, ça n'accède pas au rang de peinture. ${ }^{3}$

Si la possibilité de l'emprunt dénote la compatibilité partielle des réglementations académiques de l'image européenne et chinoise, le rejet lapidaire et définitif de l'ensemble de la production des jésuites sur le seul critère de la touche picturale révèle plus que ce qu'en comprend Zou Yigui. L'académisme occidental n'est injustement rétrogradé au rang de bricolage tâtonnant, dépourvu de maitrise et incapable de virtuosité, que parce que la peinture de cour chinoise, son homologue, faute de percevoir avec la même acuité les frontières critiques et les frontières d'usages nationaux, est influencée, sur le point crucial de la technique du pinceau, par une pratique lettrée qui rejette pourtant l'ensemble de son bagage réglementaire, y compris sa touche forcément délicate et minutieuse. À l'évidence, les protagonistes du débat ne sont pas les meilleurs juges du partage entre l'application des règles, au service de l'excellence du travail bien fait, et le jeu avec les règles, en tant qu'exercice de liberté centré sur lui-même. C'est une leçon qui donne à réfléchir sur le positivisme dont fait souvent preuve l'histoire de l'art dans son approche des sources.

\section{L'erreur ponctuelle pendant la période maoïste - enjeux historiographiques}

Cette confusion des deux morales de l'image dans l'esprit de l'ensemble des acteurs du milieu de l'art trouve son illustration la plus instructive de la période maoïste dans la réalisation puis dans la réception d'une peinture de Shi Lu (1919-1982). Issu d'un milieu favorisé, formé à la peinture lettrée, Shi Lu 
avait très tôt rejoint le camp communiste par conviction et s'était beaucoup consacré à des œuvres qu'il est d'usage de qualifier d'œuvres de propagande et qui incarnaient assez bien une forme spontanée de réalisme socialiste empreinte de techniques typiquement chinoises, notamment la gravure sur bois, ce qui lui avait valu la reconnaissance officielle. La théorisation des principes régissant les différentes imageries n'ayant jamais été très pertinente ni même très intelligible, l'appréciation se faisait en réalité à l'instinct : bien entendu, une peinture abstraite ou honteusement contre-révolutionnaire ne pouvait pas passer, mais des ouvres parfois ambiguës sur des détails dont la conscience échappait au plus grand nombre, à commencer par leurs auteurs, sont loin d'être rares. La formalisation de l'enseignement académique a mis du temps avant de porter ses fruits : Maximov n'est venu aider les meilleurs peintres chinois à se perfectionner qu'en 1955, année du départ pour Léningrad de Luo Gongliu, qui ne donnera ses cours de peinture d'histoire quà partir de 1961. C'est dans ce contexte qu'en 1959, pendant le Grand Bond en Avant, Shi Lu, avait reçu une commande officielle du Musée de l'Histoire de la Révolution et s'était rendu à Pékin pour peindre Pendant la guerre à travers le Nord du Shaanxi, en souvenir d'affrontements entre les armées communistes menées par Mao Zedong et leurs adversaires nationalistes. L'œuvre avait obtenu un grand succès public et critique, jusqu'au jour de 1964, cinq ans plus tard, où un général de l'Armée Populaire de Libération en visite au musée avait diagnostiqué une lourde erreur politique dans sa conception même, si bien que la peinture avait été décrochée et que le public n'a pu la revoir qu'en 1979.

C'est à juste titre que le corpus de règles auquel se réfère l'imagerie maoïste, comme l'imagerie soviétique, se voit attribuer l'étiquette de réalisme socialiste qui conviendrait aussi bien à d'autres imageries pourtant farouchement antibolchéviques. En comparaison de la majorité des courants de l'art moderne qu'il entendait étouffer, l'art officiel chinois était bien fondé sur une technique réaliste, mise au service de ce qu'elle était chargée de donner à voir, avec maîtrise, précision et efficacité, mais aussi avec discrétion : la technique devait s'effacer devant l'objet de la représentation. Les thèmes que la peinture devait soumettre au regard et à la réflexion étaient d'emblée restreints à la société en cours de construction, les éléments extérieurs et hostiles en étant éliminés ou, dans de rares cas, cantonnés au rôle de fairevaloir de la nouvelle société. Il s'agit donc bien d'un réalisme social, auquel il ne manque plus que l'exaltation de ladite société pour justifier le passage de "social " à "socialiste " et retomber sur une définition rigoureusement identique à celle de la peinture de cour qui prévalait sous l'Empire. Or le suffixe est bien justifié par la prégnance d'un arsenal de procédés visant à rendre sensible la grandeur de la société représentée. Parmi ces artifices, peu nombreux et assez simples, l'usage extrêmement répandu de la contreplongée s’impose par son efficacité redoutable, comme en témoignent Les cinq braves 
du Mont Langya de Zhan Jianjun en 1959, la Conférence de Gutian de He Kongde en 1972, Monter la garde pour notre grande patrie de Shen Jiawei en 1974 et tant d'autres peintures extrêmement célèbres. La rupture d'échelle entre le premier plan et l'arrière-plan rend encore plus impressionnante la figure centrale de la peinture, de Mao à Lushan de Jin Shangyi en 1966 et du Président Mao se rendant à Anyuan de Liu Chunhua la même année au Mao Zedong à Jinggangshan peint par Luo Gongliu en 1962. Ces procédés sont souvent combinés dans la même toile, ce qui rend l'effet plus saisissant, tandis qu'un troisième artifice suggère d'exploiter l'environnement immédiat du personnage magnifié, afin de susciter des rapprochements symboliques aussi percutants que faciles à comprendre : la tête du jeune Mao Zedong en route pour Anyuan, la mine déterminée, est nimbée de nuages qui lui tracent son chemin ; dans la Conférence de Gutian, elle est alignée avec des portraits de Marx et de Lénine accrochés au mur derrière l'orateur ; lorsque Mao, assis et pensif, fume une cigarette dans la peinture de Luo Gongliu, la montagne derrière lui amplifie sa silhouette et conforte sa stature historique inébranlable.

Ces procédés ne nécessitent pas pour autant la connaissance des méthodes ou des théories soviétiques dont l'ignorance excuserait d'éventuelles fautes commises par les peintres, car il s'agit de formules auxquelles se range spontanément tout professionnel de l'image raisonnablement inventif cherchant à exalter de manière appuyée une histoire ou une figure particulière. Le Chateaubriand de Girodet (1809), l'Officier de chasseurs à cheval de la garde impériale de Géricault (1812), le Bonaparte franchissant le GrandSaint-Bernard de David (1801), le Voyageur contemplant une mer de nuages de Friedrich (1818) ignoraient tout du communisme, mais les procédés sont identiques. Les gravures sur bois chinoises, considérées comme " expressionnistes ", des années 1930-1940 ne sont certes pas sans lien avec l'activisme du Parti communiste chinois, mais il est difficile d'en dire autant du $\mathrm{Nu}$ de Zhou Bichu en 1932... Que les mêmes procédés se retrouvent chez des " néoclassiques " français, des " romantiques » allemands, des bolchéviques, des nazis, des fascistes, mais aussi de doux rêveurs chinois, des journalistes ou des publicitaires contemporains de tous pays, montre bien qu'il ne s'agit pas d'appliquer un cahier des charges imposé par une "idéologie " - mot commode - mais d'exprimer une intention par ce qu'on s'autorise ou non dans la confection de l'image. L'application d'une formule type liée à une propagande étatique n'est qu'un cas de figure possible; le plaisir pris à la confection d'une image forte éventuellement impliquée dans une tradition artistique ou iconographique en est un autre, a priori beaucoup plus général.

En peignant Pendant la guerre à travers le Nord du Shaanxi, Shi Lu ne pensait certainement pas nuire à la propagande du Parti et croyait sans doute même la servir, mais il voulait surtout faire œuvre, dans la lignée des deux patrons qu'il s'était donnés en se choisissant son pseudonyme, deux personnalités aussi indé- 
pendantes qu'engagées, le peintre Shi Tao du début de la dynastie des Qing, lettré s'il en fut, et l'écrivain moderne Lu Xun, compagnon de route turbulent et inclassable du Parti. Bien qu'il ne s'agisse pas d'une peinture à l'huile, l'œuvre de Shi Lu donne au premier coup d'œil des gages dignes des toiles les plus orthodoxes. La perspective est bien une forme de contre-plongée, dans l'esprit de la peinture chinoise classique ; la perte de la notion d'échelle qu'inspire le paysage montagneux donne bien à voir un Mao Zedong se mesurant à l'immensité ; et la recherche d'un symbolisme politique de cette image peut parfaitement déboucher sur un discours à la gloire du grand homme visionnaire, qui parvient à faire basculer le destin de tout un pays par la seule force de ses convictions. Mais le problème avec les interprétations symboliques, c'est que chacun a la sienne et qu'aucune n'a le dernier mot sur les autres. Le rôle des experts soviétiques n'a jamais été d'apporter aux Chinois ce qu'ils avaient déjà, mais plutôt de leur assurer la maîtrise absolue, jusque dans ses ultimes conséquences, de cette technique davantage rodée à Léningrad qu’à Pékin, de façon que l'œuvre elle-même rende impossible toute perception erronée non conforme à la morale dont elle était l'expression. En dépit de ses qualités artistiques et de ses bonnes intentions politiques, il n'est pas faux de voir dans la peinture de Shi Lu un Mao Zedong statique, isolé, inexpressif, au bord du précipice, dont tous les horizons semblent irrémédiablement bouchés... Laisser la moindre prise à une telle interprétation, comme l'avait fait Shi Lu sans s'en rendre compte, était incontestablement une erreur du point de vue d'un réalisme socialiste canonique. Mais le fait qu'une telle erreur n'ait été perçue ni par les spécialistes les plus autorisés, commanditaires ou critiques, ni par le public très nombreux pendant cinq longues années montre aussi que cette peinture, en dépit des discours convenus, avait été perçue comme une œuvre d'art dans le sens hérité de la tradition de la peinture lettrée et non comme un chef-d'œuvre académique dans la veine "réaliste socialiste " propre à l'époque. La faute n'était pas moindre, au contraire, et l'épilogue montre la portée artistique paradoxale de ces erreurs. Brisé par ce revirement incompréhensible puis par les persécutions subies pendant la Révolution culturelle, Shi Lu devait sombrer dans la schizophrénie et c'est dans cet état psychologique, peu propice à l'application des règles codifiées qu'il prenait déjà de haut par le passé, qu'il créera au soir de sa vie ses plus belles œuvres, si éloignées du réalisme socialiste qu'elles ont pu être associées à l'émergence du courant de la « nouvelle peinture lettrée » au début des années 1980.

\section{L'erreur intégrée - enjeux artistiques au-delà de la période maoïste}

La carrière de Shi Lu correspond assez bien à la vulgate que l'histoire de l'art de la période maoïste aime à donner du devenir des artistes de renom dans la société communiste : sommés de se plier à la conception utilitariste 
d'une imagerie au service de la propagande, ils n'avaient d'autre choix que de se conformer au modèle, ce qui ne devait malheureusement pas les mettre à l'abri des persécutions sous la Révolution culturelle. Cette vision des choses n'est évidemment pas fausse, mais elle occulte le phénomène artistique majeur de l'époque et ne rend pas bien compte de la réalité partielle à laquelle elle s'attache. Que montrent exactement les photographies de peintres envoyés à la campagne, comme celles de Wu Zuoren, Ye Qianyu et Jiang Zhaohe réalisant des peintures murales dans un village du Hebei en 1958 ? Certes, il est évident que ces peintures simplistes ne reflètent pas leurs véritables centres d'intérêt artistiques, mais cette corvée de courte durée n'affecte en rien leurs aspirations ni la maîtrise de leur art qui lui survivront. Il faudrait se demander s'il en va de même pour les enfants de paysans qui les regardent avec curiosité et qui seront beaucoup plus perméables aux projets de refonte culturelle nourris à leur égard par le Parti, bel et bien secondé, bon gré mal gré, par les peintres célèbres ou moins célèbres envoyés sur le terrain.

Il est toujours possible pour un artiste de tirer profit d'une contrainte; un poète un tantinet joueur s'accommode avec plaisir du gage de la rime imposée. Très tôt, des peintres de l'école du Lingnan, Gao Jianfu en tête, se sont fait fort d'intégrer des nouveautés incongrues à leurs peintures : une escadrille d'avions de chasse dans le ciel d'un paysage n'a pas moins d'intérêt pictural qu'une nuée d'oiseaux. Ce serait faire preuve d'un conservatisme exagéré que d'y voir une " erreur ", ou plutôt il n'y aurait "erreur " d'un point de vue artistique que si l'impératif de la nouveauté était une règle positive et non l'un des ingrédients possibles d'une combinaison de choix qui ne valent que relativement les uns aux autres dans une œuvre particulière. Un cadrage serré sur la tête de pylônes à haute tension dont les câbles partent en tous sens et déchirent le paysage en arrière-plan peut constituer un prodigieux terrain d'expérimentation graphique pour un artiste comme Fu Baoshi en 1961 : l'effet d'ensemble produit par la peinture s'avère pourtant moins flatteur que le terme " abondance " employé dans le titre ne le laisse prudemment croire. L'ironie des contrastes donnés à voir pour ce qu' ils sont sait se faire très discrète, de même que l'humour des décalages faussement résorbés peut déstabiliser les normes les moins ouvertement discutables. Le vieux lettré chinois a beau être réputé courtois et conciliant, si on lui demande d'adapter son art à une réglementation qui en fait fi au nom du principe de l'expertise (专 zhuān) combinée au "rouge " de l'enthousiasme révolutionnaire (红 hóng) - ce qui revient tout bonnement, en matière picturale, au réalisme (expert) socialiste (rouge) - il n'est pas à exclure que, trahi par ses facultés limitées, il commette l'erreur de croire qu'on lui demande d'agrémenter le jeune bambou qu'il s'apprêtait à peindre d'une «brique rouge " (红砖 hóngzhuān) qui l'écrase mais n'empêche pas ce symbole de résistance dans l'adversité de rebiquer vers le ciel. C'est du moins la bévue qu'a commise Wu Hufan en 1959, «s'appliquant modestement 
à l'étude de la ligne expert et rouge " qu'on prétendait lui imposer. L'erreur est incontestablement de son fait et il devait la payer cher quelques années plus tard pendant la Révolution culturelle. Elle devait pourtant changer de camp à l'occasion du succès public de l'audacieuse peinture qu'il avait consacrée en 1965 au champignon atomique marquant l'entrée de la Chine dans le club des puissances nucléaires. "Célébrons le succès de l'explosion de notre bombe atomique nationale! » calligraphiait-il en vis-à-vis de l'exercice de style virtuose dont le motif lui procurait le prétexte, en plus du plaisir du quiproquo.

À la différence des artistes patentés, les paysans n'étaient pas présumés rétifs aux normes picturales du réalisme socialiste, mais ils en étaient de fait beaucoup plus éloignés encore, tant par leur propre morale de l'image que par leurs compétences techniques ou par l'iconographie traditionnelle de ce qu'il est convenu de traduire par " peinture de calendrier ». La signification littérale du terme niánhuà, " peinture annuelle ", indique clairement le caractère jetable de cette imagerie, propitiatoire par ses thèmes et marquée par l'occasion de sa confection (nouvel an, fêtes...), qui recourt à des techniques traditionnelles d'une longévité limitée, à l'instar des papiers découpés ou des sentences parallèles collées sur les portes extérieures. Pour ne rien arranger, les paysans étaient la véritable cible des attentions et des réformes souhaitées par le régime. S'il est de bon ton d'insister sur la consigne donnée aux artistes de se rapprocher des paysans et de se conformer à leurs goûts, il faut néanmoins se rendre à l'évidence que la conséquence réelle et durable de cette politique a été d'instiller dans l'imagerie paysanne des thèmes et des techniques du réalisme socialiste qui, ne correspondant ni aux aspirations imagières ni aux moyens techniques des populations, les dénaturaient. Les bébés joufflus au milieu de corbeilles de fruits passent beaucoup moins bien dans une peinture à prétention réaliste que dans un découpage monochrome, simplifié et stylisé ; les vues plongeantes sur des usines, des écoles ou des exploitations agricoles, n'ont pas leur place collées à la fenêtre de la cuisine et sont truffées d'erreurs de perspective ou de naïvetés ; les peintures de propagande, enfantines, perdent la solennité qui leur sied et donnent lieu à des remplissages de couleur parfois rose vif à relents impressionnistes. Avec le temps, cette imagerie s'est étoffée de photographies de plats roboratifs aux couleurs criardes destinées à occuper la moitié du mur de la salle à manger et d'autres joyeusetés du même acabit. Forcée de se prendre au sérieux, la culture visuelle paysanne, jusqu'alors originale, spontanée et sans prétention, s'est retrouvée radicalement transformée par cette cascade d'erreurs et de fautes de goût ; compte tenu du caractère essentiellement rural du pays, c'est toute la Chine qui en a été affectée.

L'histoire de l'art contemporain a beaucoup de mal à suivre un autre modèle de raisonnement que celui de l'influence, qui a l'avantage non négligeable de toujours placer l'Occident à l'origine de tout, mais aussi de mobiliser très peu de concepts à force de les recycler, ce qui est appréciable, et, par 
un raisonnement d'une circularité remarquable, d'en dissimuler la faiblesse en imputant leur inefficacité parfois flagrante aux aléas de leur transplantation, avec cet argument imparable que les prétendus copieurs ne seraient pas à la hauteur du paradigme. Ainsi, l'art chinois des années 1990 serait kitsch, sans les qualités du kitsch original cela va de soi, par imitation de l'art pop occidental lancé à la conquête du monde selon une logique qui n’a pas à être discutée et ne laisse aucune place à l'erreur. Le dévoiement de l'imagerie paysanne pendant la période maoïste qui a vu naître et grandir les artistes chinois concernés pèse-t-il le moindre poids face au modèle universel ? Quant au fait que depuis la peinture lettrée, l'art chinois, traditionnel, moderne ou contemporain, s'approprie par jeu la réglementation de l'image dont il se démarque radicalement sur le fond, il n'est pas non plus de l'ordre du pensable, tel que l'ont défini les catégories primitives, ce qui clôt le débat. Les matériaux utilisés ne sauraient davantage constituer un indice de quoi que ce soit : peu importe que les frères Luo utilisent de la laque (ils sont du reste priés de s'appeler les Luo brothers), que les peintures de Liu Zheng soient des rideaux de perles en plastique, que les toiles de Chang Xugong soient réalisées en broderies d'un clinquant insoutenable, que $\mathrm{Hu}$ Xiangdong soit obsédé par la peinture de nourriture et de culturistes, ce n'est que diversification mercantile de la matrice occidentale. Quand le critique Li Xianting expose en détail la raison d'être de ce courant artistique du milieu des années 1990 dans divers catalogue d'expositions ${ }^{4}$, bien mal lui prend de consacrer une partie de son raisonnement à une comparaison avec le courant kitsch dans ses versions étrangères, car l'explication par la contiguïté se passe très bien des complications d'une analyse de fond dès lors vouée aux oubliettes. Il n'en reste pas moins que l'art criard n'est pas le produit d'une influence extérieure, mais le résultat artistique d'une série d'erreurs et de corrections, puis de détournements de ces erreurs et de ces corrections, qui ont fait boule de neige.

\section{De l'intérêt artistique de l'erreur}

C'est ainsi que des œuvres, des carrières et des courants artistiques ont trouvé tantôt leur origine accidentelle, tantôt leur source d'inspiration entretenue et amplifiée, dans les erreurs inévitablement provoquées par la confrontation de leur morale artistique à d'autres traditions imagières, la principale étant la réglementation en vigueur à la cour impériale, d'abord récupérée par le régime communiste sous le nom de réalisme socialiste puis prolongée, sitôt les écoles d'art rouvertes à la fin de la Révolution culturelle, par le rigoureux académisme, un rien pompier, enseigné à travers tout le pays dans le cadre de

4 Li Xianting, "Une imitation sarcastique du goût paysan nouveau-riche. L'art criard et son contexte ", catalogue de l'exposition "Alors, la Chine ? ", Paris, Centre Pompidou, 2003, p. 104-113. 
ces institutions. Cette dernière étape de la transition est la mieux documentée et donc la moins contestable, car les premières gloires du marché chinois n'étaient autres que les grandes figures de la peinture d'histoire réaliste socialiste, notamment Chen Yifei, reconverti dans l'évocation érotisée d'une atmosphère shanghaienne mythifiée, et Jin Shangyi, grand portraitiste, non plus du président Mao, mais de jeunes filles pensives, belles et nues de préférence. Le renversement qu'a constitué l'implantation progressive de l'art contemporain au sein des institutions académiques, seule issue sociale pour les jeunes artistes des années 1980, peut lui-même être décrit comme un insidieux grignotage par l'erreur de leur corpus réglementaire, débouchant, chaque fois au prix de débats et de rectifications sévères, sur l'émergence successive de l'art de la cicatrice et de l'art du terroir. Ces derniers prépareraient à leur tour le terrain aux dérives fauvistes et impressionnistes qui devaient mener jusqu'à la peinture abstraite, avant que divers bricolages, installations ou performances remettent finalement en cause l'identification naïve de l'art à l'image, renouant dans les faits - mais pas dans le discours, loin s'en faut - avec la nature exclusivement critique de la peinture lettrée, attestée dès ses balbutiements par nombre de bizarreries comportementales de ses artistes emblématiques, que leurs historiographes ont relevées sans en tirer la moindre conclusion théorique, animés qu'ils étaient par le seul souci scolaire de l'exhaustivité biographique. Dans cette longue reconquête, chaque nouvelle erreur n'était que partielle et amendable, donc excusable, car se tromper, même avec insistance, n'est pas agresser ; mais il est vrai que la direction des établissements était peuplée de grands anciens, bien moins hostiles au retour sur le devant de la scène d'une pratique authentiquement artistique, même incomprise, que ne l'autorisaient officiellement leurs fonctions.

Quand bien même l'erreur est avérée dans l'esprit des censeurs, cette erreur pour autrui n'est guère qu'une facette banale du mécanisme anthropologique de la divergence, préalable nécessaire à la négociation de l'acceptation mutuelle qui fait la vie sociale. Il en va tout autrement quand l'erreur constitue le cœur même de l'art, comme dans les œuvres de Xu Bing à partir de la deuxième moitié des années 1980. La réalisation du Miroir pour l'analyse du monde, également dénommé le Livre du Ciel, a coûté à Xu Bing plusieurs années passées à concevoir et à graver - à l'envers, est-il nécessaire de le préciser? - des milliers de caractères chinois qui n'existent pas et avec lesquels il se préparait à imprimer son grimoire exposé sous la forme d'une imposante installation. Chaque caractère inventé l'était à partir d'authentiques composants de caractère chinois et la structure du caractère comme les modalités de combinaison des composants étaient également conformes aux modes d'agencement propres à l'écriture chinoise. Mais aucun de ces caractères ne notait un mot de la langue et n'était pourvu d'une quelconque signification, à l'exception d'une poignée d'erreurs dans l'erreur, décelées, paraît-il, par 
des érudits spécialistes d'antiques variantes graphiques inusitées et devenues indéchiffrables. Quelques années plus tard, Xu Bing, persistant dans l'erreur, avait mis en scène deux cochons, un mâle et une femelle, l'un tamponné de ses faux caractères chinois, l'autre de graphies tout aussi stériles résultant de la combinaison aléatoire de lettres latines, ce qui ne devait cependant pas empêcher les deux Animaux culturels de faire ensemble ce qu'ils avaient à faire. La première réaction de la critique, en un sens justifiée, était de souligner la magistrale absurdité de la besogne que s'était infligée Xu Bing, mais cette sidération s'accompagnait souvent de véritables bouffées de rage qui, passée l'énumération des quelques proverbes adéquats, tentaient de se donner une caution théorique dans des tirades stéréotypées sur la trahison de la culture chinoise et l'influence délétère de l'Occident. Reconnaissant sans doute qu'il ne valait pas mieux qu'un aveugle cherchant à attraper un corbeau dans le noir ou qu'un fantôme croyant pouvoir se guider dans la nuit en tâtonnant une muraille dont il ne s'aperçoit pas qu'elle est circulaire, Xu Bing, assisté d'une petite équipe, avait alors passé une vingtaine de jours à tamponner l'intégralité d'un beau tronçon de la Grande Muraille, qu'il devait exposer sous le titre Fantôme tapotant la muraille.

Il est tout de même bien léger d'attribuer la paternité du sentiment de l'absurde à une prétendue modernité occidentale, alors qu'il s'agit de l'une des principales possibilités offertes par le jeu critique à l'origine de la tradition artistique chinoise - sans même parler de l'expérience quotidienne de chacun. L'accumulation disproportionnée de restrictions et de frustrations entrant en résonnance les unes avec les autres dans la confection d'une ouvre est certes absurde, mais c'est ce qui fait la beauté aride des paysages de Ni Zan, tendus vers cet extrême déjà sous les Yuan (1279-1368) ; à l'inverse, c'est l'accumulation des prises de liberté qui procure le plaisir de la provocation et, n'était son humour, l'art kitsch n'en est pas loin ; créer, dans un même travail, un contraste ouvertement inconciliable entre des choix marqués par la transgression et d'autres empreints de réticence, c'est faire œuvre d'ironie, dans une mouvance où, en dépit de la variété des configurations plastiques, artistiquement bien secondaires faut-il le répéter, le pop politique et le réalisme cynique des années 1990 peuvent rejoindre les malices d'un Qi Baishi (1864-1957); en revanche, lorsque la confrontation des contraires est assumée comme une évidence naturelle et neutre, c'est d'humour qu'il s'agit. Les œuvres de Xu Bing ou de Wu Hufan trouvent leur juste place dans cette catégorie, même si l'humour du premier tend vers l'absurde et si celui dont a fait preuve son aîné frisait la provocation. Le plaisir pris à inclure dans leurs œuvres l'acceptation de la critique adverse, en dépit de l'aberration flagrante de leurs actes à l'aune de ses critères, montre qu'artistiquement l'erreur est l'autre nom de l'humour, mais rien n'est plus difficile que de l'expliquer aux partisans exclusifs de l'académisme, surtout s'ils s'ignorent... 


\section{Mécanisme et diversité de l'erreur}

L'exploitation systématique de l'erreur comme matière artistique chez certains artistes permet de préciser ce que recouvre en art la notion si floue d'erreur et le terrain le plus propice se trouve chez ceux qui se réclament d'un rationalisme sans concession, à l'instar des artistes du " traitement froid " pendant la Nouvelle Vague du milieu des années 1980, autour de Zhang Peili et Geng Jianyi, ou du groupe de la Nouvelle Mesure (Xìn kèdù) incarné par Wang Luyan, Gu Dexin et Chen Shaoping au début des années 1990. À l'occasion d'une exposition organisée au Centre d'Art contemporain de Santa Monica à Barcelone en 1995, ces derniers ont réalisé deux œuvres sur un même protocole rigoureux, délibéré collectivement. Pour la première, ils avaient créé une grille à laquelle ils avaient intégré le texte en catalan de la préface du catalogue, de façon à pouvoir opérer une sélection de cases à conserver ou à rejeter selon des règles redéfinies en commun à chaque application de la grille : l'œuvre finale ne laissait qu'une juxtaposition de cinq mots catalans sans le moindre lien donc la moindre signification. La même méthode a ensuite été appliquée aux photographies d'identité des trois membres du groupe, qui au fil des découpages et des recombinaisons se sont réduites à cinq cases minuscules de diverses nuances grisées alignées au milieu d'une page blanche. La rationalité technique, mise en œuvre avec méticulosité, aboutissait à une production formalisée mais inepte et le langage se montrait incapable de rien désigner ; quant à la rationalité sociologique de la négociation méthodique entre les membres du groupe, elle ne faisait que contribuer à ce résultat proche du néant. De la raison naissait l'erreur, mais ce n'était pas sans un certain plaisir. Forcé, comme tous les artistes participant à « Magiciens de la Terre » en 1989, de s'essayer à une brève définition de l'art pour le catalogue de l'exposition, Gu Dexin, dont le laconisme notoire confine au mutisme, avait fait preuve d'une concision lumineuse et prophétique : "L'art, c'est ce qui est tiré de l'expérience d'une recherche de plaisir dans des activités très compliquées $"^{5}$.

L'erreur est donc la concrétisation du décalage que la culture introduit dans le rapport naturel au monde. La raison tourne toujours un peu à vide et le Miroir pour l'analyse du monde de Xu Bing jouait déjà de cette marge d'erreur poussée jusqu'à l'absurde : la colossale dépense de technicité de son écriture ne produisait ni mot ni sens et le langage restait hors de portée. Ce grossissement de l'erreur est aussi illustré par les célèbres Vélos réformés de Wang Luyan dont le pédalier trafiqué fait rouler vers l'arrière quand on pédale vers l'avant. Réflexion faite, le procédé est à peine plus contre-nature au regard de sa fin escomptée que le simple glissement de la marche à la gestuelle du pédalage, de même que si la réitération à l'infini d'une photocopie de photocopie, 
également attestée dans l'art contemporain chinois, finit par rendre méconnaissable l'image de départ, c'est que la technique marquait déjà du sceau de l'erreur la première reproduction. Tout un pan de l'histoire de l'art le souligne a contrario en rendant inopérant ce mécanisme rationnel de l'erreur artisanale, qui se voit supplanté par une instrumentation non technique de la donne naturelle ou d'objets traités comme tels, dans des pratiques dont la bigarrure terminologique masque l'unicité, de l'ancestrale sculpture de racine (gèndiāo) aux installations in situ et autres ready made, mono ha ou land art. Le jeu de langage austère accompagnant la sculpture indéterminée de Wang Luyan sur le thème Gauche/Droite montre que la rationalité logique n'est pas épargnée, ce que confirme, même s'il est plus complexe, le cas de la lecture, filmée par Zhang Peili, de l'article "Eau " d'un dictionnaire de référence, confiée à une présentatrice de la télévision nationale. L'inintérêt du contenu, familier de la peinture lettrée, entre en résonnance avec une prononciation d'une correction surhumaine pour l'oreille sudiste de l'auteur, au mandarin bien moins pur, mais le fossé linguistique s'y double d'une divergence sociologique, plus sensible encore dans les jeux de traduction du chinois à l'anglais et inversement, qui n'ont pas besoin de beaucoup d'allers-retours pour tourner au cadavre exquis sur le principe de l'erreur cumulative.

C'est donc à plus d'un titre que l'erreur peut jouer un rôle majeur à la source même de l'œuvre et l'analyse des procédés entremêlés que cache l'unicité du mot serait un bon moyen de remettre de l'ordre dans les catégories usuelles de l'histoire de l'art, dont le flou et la polysémie constituent plus qu'une entrave à la compréhension des phénomènes en jeu, bien au-delà du cas chinois.

Frédéric Le Gouriérec EA 4270 CRIHAM

Université de Poitiers

frederic.le.gourierec@univ-poitiers.fr

\section{Résumé}

La notion d'erreur n'a pas de pertinence dans la tradition artistique chinoise. Seule la confrontation entre l'imagerie réglementée de la cour et l'imagerie artistique de la peinture lettrée a fait naitre un problème que devaient amplifier leurs avatars successifs, de la tentative du réalisme socialiste de s'annexer l'art dit moderne à la confrontation entre l'institution académique et l'art contemporain. Cette perspective historique ne dispense pourtant pas d'une analyse des mécanismes de l'erreur dont il s'avère qu'ils n'ont d'unité que dans le mot.

\section{Mots-clés}

Art contemporain chinois, peinture lettrée, réalisme socialiste, peintres missionnaires jésuites, art criard (yansu yishu). 


\section{Abstract}

The notion of error is not relevant in the Chinese artistic tradition. It is merely through the confrontation of imperial court imagery requirements with the artistic freedom of literati painting, that some issues around this question arose. These ones were amplified by successive adversities between both traditions, such as the attempt of Socialist Realism to monopolize the so-called Modern Art, or later on the confrontation between the academic institution and contemporary art. In any case, this historical perspective means no exemption from analyzing the mechanisms of the error itself, which seem to have no unity but within the wording.

\section{Keywords}

Chinese contemporary art, literati painting, socialist realism, jesuit missionary painters, gaudy art (yansu yishu). 